\title{
Research on improving measurement accuracy of acoustic transfer function of underwater vehicle
}

\author{
Jiangqiao $\mathrm{Li}^{1,2, *}$, Li Jiang ${ }^{1,2}$, Fujian $\mathrm{Yu}^{1,2}$, Ye Zhang ${ }^{1,2}$, and Kun $\mathrm{Gao}^{1,2}$ \\ ${ }^{1}$ Systems Engineering Research Institute, Fengxian east road No.1,Haidian district,Beijing, China \\ ${ }^{2}$ Science and Technology on Underwater Acoustic Antagonizing Laboratory, Fengxian east road \\ No.1,Haidian district,Beijing, China
}

\begin{abstract}
To address the problem that acoustic transfer functions with underwater platforms cannot be measured accurately, this paper presents a method based on phase compensation to improve the accuracy of acoustic transfer function measurements on underwater platforms. The time-domain impulse response signals with multiple cycles are first collected and intercepted, and then their phase differences are estimated using the leastsquares method, and phase compensation is used to align the phases of all the signals, and then the impulse response signals are weighted and averaged over all the impulse response signals to cancel out the random noise. The water pool test proves that this method reduces the measurement random noise while obtaining a high-fidelity time domain transfer function, which effectively improves the signal-to-noise ratio of the measurement. The method adopts only one measurement signal, and without changing the measurement system, the random noise is cancelled out by the in-phase superposition of the multi-cycle impulse response signals to avoid the nonlinear distortion of the measurement results.
\end{abstract}

\section{Introductory}

Hydroacoustic signals received by a hydrophone array installed on the port side of a submerged platform consist of the following components: the first is the direct acoustic signal, and the second is the scattered acoustic signal (including reflections and bypasses) formed by the direct acoustic action on the platform. As the hydrophone is close to the platform surface, the frequency bands of the two signals are close to each other, so the second type of signal is a kind of interference relative to the direct acoustic signal, making the waveform and characteristics distorted, the most obvious and typical effect is that the directionality of the receiving hydrophone or the base array will be changed, which will affect the performance of the subsequent signal processing. Knowledge of this platform acoustic scattering characteristic is the basis for the overall performance evaluation as well as the acoustic compatibility performance assessment.

\footnotetext{
*Corresponding author: lijiangqiao11@163.com
} 
Due to the UUV fuselage, the physical process of acoustic signal scattering during propagation from the sound source to the hydrophone can be modeled as a linear timeinvariant acoustic filter system, which is fully characterized by the transfer function of the system [1]. It is the acoustic scattering problem in underwater platforms that can be described using the acoustic transfer function.

\section{Underwater platform sound scattering characteristics study}

The study of sound scattering from simple targets to complex structural targets has been the focus of research, and a large number of studies have been carried out in theoretical prediction models, numerical calculations and experimental measurements, and a large number of model scaling experiments and marine measurements have also been conducted, and rich measurement data have been obtained.

The numerical calculations based on the scaled-down model mainly adopt the finite element method for sound scattering calculations, but the finite element method faces great difficulties in calculating the sound scattering characteristics of complex underwater platforms, especially for large scale platforms, the calculation volume is intolerable and even impossible to complete when calculating their scattering in the slightly higher frequency band ${ }^{[2]}$. Therefore, the acoustic transfer function of underwater platforms is mainly obtained through experimental measurements.

The study of sound scattering from simple targets to complex structure submarine targets has been the focus of foreign research, has formed a complete research and experimental system, especially for underwater target high-frequency sound scattering research has made important progress, countries have developed corresponding calculation models and software, such as Sweden's SUBTAS (submarine target intensity prediction) software; the United States submarine sound scattering numerical simulation software TRANAIR; the United Kingdom's SIGNATURES software; the French submarine highlights model, and so on.

\section{A phase-compensation based method to improve the signal- to-noise ratio of transfer function measurements}

In conventional measurement experiments, in order to ensure the accuracy of measurement, a specific test signal, such as the longest pseudo-random sequence (MLS), exponential sweep sequence, etc., is emitted from the sound source, and the transfer function can be obtained by correlating or convolving the signal recorded by the hydrophone with the emitted signal. However, in the actual measurement of acoustic transfer function on an underwater vehicle, the noise of the measurement environment and the inherent noise of the transmitting and receiving system (transmitting transducer, receiving hydrophone, power amplifier, etc.) will inevitably interfere with the measurement. The signal-to-noise ratio of the measurement system can be effectively improved by increasing the power of the test signal, but the measurement system is usually limited by various aspects, such as the transmitting power limit of the transmitting transducer, or the nonlinear distortion caused by the signal source amplitude exceeding a certain dynamic range. Therefore, in order to improve the measurement results, this paper proposes a phase-compensation based method to improve the SNR of acoustic transfer function measurements on underwater vehicles. 


\subsection{MLS measurement transfer function methods}

Assuming that the measurement system is a linear time-invariant system, the circular autocorrelation function for an MLS sequence $x(n)$ of length $\mathrm{L}_{\text {is }}{ }^{\left[{ }^{-5}\right]}$ :

$$
\phi_{\mathrm{xx}}(n)=x \operatorname{corr}(x(n))=\sum_{k=0}^{k=L-1} x(k) x(n+k)_{L}=x(n) \otimes x(-n)=\left\{\begin{array}{l}
L, n=0 \\
-1, n \neq 0
\end{array}\right.
$$

The above property shows that the autocorrelation function of $x(n)$ approximates an impulse signal if it is divided by the length of the sequence. Using the above properties of MLS, we can derive the principle of the MLS sequence measurement transfer function:

$$
\begin{aligned}
y(n)=h(n) \otimes x(n) \\
\hat{h}(n)=\frac{1}{L} \phi_{x y}(n)=\frac{1}{L} x \operatorname{corr}[x(n), y(n)]=\frac{1}{L}[y(n) \otimes x(-n)] \\
=\frac{1}{L}\{[h(n) \otimes x(n)] \otimes x(-n)\}=\frac{1}{L}\{h(n) \otimes[x(n) \otimes x(-n)]\} \\
=h(n) \otimes \frac{1}{L} \phi_{x x}=h(n)-\frac{1}{L} \sum_{k=0}^{k=L-1} h(k)
\end{aligned}
$$

where, $\hat{h}(n)$ is the transfer function obtained from the actual measurement, $(n)$ is the transfer function of the system to be measured, $x(n)$ is the MLS sequence of the input system, and $y(n)$ is the output signal obtained from the system measurement.

The output signal $y(n)$ obtained by the system is computationally correlated with the input MLS sequence $x(n)$ and divided by the sequence length $\mathrm{L}$. When the sequence length $\mathrm{L}$ is sufficient, $\frac{1}{L} \sum_{k=0}^{k=L-1} h(k)$ approximates an impulse signal. Such a method yields $\hat{h}(n)$ approximated by $(n)$. It is important to note that the length L of the MLS sequence needs to be greater than the length of the system transfer function, otherwise $x(n)$ is not equal to the circular and linear convolution in the inter-correlation, which can lead to time mixing problems. The measurement flow can be summarized in Figure 1.

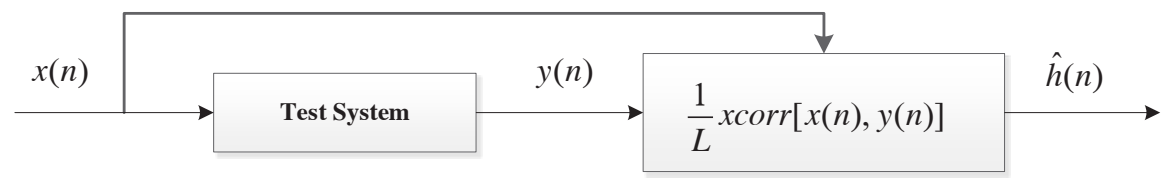

Fig. 1. Measuring transfer function with MLS.

So the process of measuring the transfer function using the MLS sequence is as follows.

(1) Generate MLS sequences $\hat{h}(n)$ of order N length $L=2^{N}-1$.

(2) Measure the output signal $y(n)$ of the resulting system.

(3) Calculate the cross-correlation between $x(n)$ and $y(n)$ and divide by the sequence length $\mathrm{L}$ to obtain $\hat{h}(n)$.

The MLS signal is stable and can be generated repeatedly; its periodicity ensures that there is no signal truncation effect during measurement; the MLS signal provides a high signal-to-noise ratio, and the measurement process is easy to implement. 


\subsection{Phase difference calculation and phase compensation}

The basic idea of the transfer function noise reduction method based on phase compensation proposed in this paper is to align the phase during each cycle of the multiperiod measurement and then average the pairwise time-domain impulse response functions to cancel out the random noise that behaves as a white spectrum in the spectrum. It is assumed that although the measurements of the transfer functions of different cycles are not aligned in terms of timestamp pairs, their time difference is consistent, so take the leastsquares estimate of this time difference, perform phase compensation, get the transfer functions of the time-aligned multi-cycle measurements, and then average them to achieve noise reduction.

The following expression can be derived from the relationship between phase difference and time.

$$
\begin{gathered}
\varphi_{1}(\omega)-\varphi_{2}(\omega)=\omega \cdot \tau \\
\omega=2 \pi f
\end{gathered}
$$

where $\varphi_{1}(\omega)$ is the phase response of the transfer function measure $\mathrm{d}$ for the first cycle, $\varphi_{2}(\omega)$ is the phase response of the HRTF measured for the second cycle, and $\tau$ is the time difference between the HRTFs measured for the first and second cycles, which is the slope of the phase response curve.

When the transfer function is measured continuously over multiple cycles, the time difference during the period is caused by the different time intervals between the sampling points due to the inconsistency between the timing equipment of the transmitting system and the timing equipment of the acquisition system. A phase-compensation method is used to make up the time difference between the cycles so that the phase of the cycles is consistent. As the stability of the transmission function is poor in the part with a low signalto-noise ratio, there is some oscillation in the phase, which affects the estimation of the slope, the slope can be obtained from the frequency point with a relatively high signal-tonoise ratio and no significant oscillation in the phase, and then phase compensation is made.

\subsection{Time-domain averaging}

The method proposed in this paper is a time-domain averaging method based on phase compensation, where a multi-cycle cycle of measurements is performed in a measurement environment to estimate and compensate for the phase deviation of the measured transfer functions, and the measured transfer functions are averaged after alignment.

The time domain averaging proposed in this paper is a technique of averaging the measurement results of each of the R-periods of the input signal separately intercepted, and then averaging them in the time domain after phase compensation of the post-R-1 band signal. It can effectively improve the signal-to-noise ratio of the measurement results, and averaging the measurement results of $\mathrm{R}$ cycles can improve the signal-to-noise ratio $\mathrm{R}$ times.

Set that the transfer function measured by the input signal with period is $y(n)$, which contains the transfer function $h(n)$ in the absence of systematic noise and the random noise $e(n)$ present in the measurement system, as follows:

$$
y(n)=h(n)+e(n)
$$

Intercepting the periodic lengths into R segments, the noise free system function is $\mathrm{h}$ for each segment, while the system noise $\mathrm{n}$ for each segment is uncorrelated. 
Intercepting $y(n)$ into $\mathrm{R}$ segment $y_{i}(n)$ by period length, the system function under noise-free conditions for each segment is $h_{0}(n)$, while the system noise $e_{i}(n)$ for each segment is uncorrelated.

$$
y_{i}(n)=h_{0}(n)+e_{i}(n), i=1,2, \ldots, . R
$$

This sums and then averages each segment $y_{i}(n)$, while the expected value of random noise is 0 . As $\mathrm{R}$ increases $h_{i}(n) \approx h_{0}(n)$, the transfer function for noise reduction is obtained:

$$
\begin{array}{r}
h^{\prime}(n)=\frac{1}{R} \sum_{i=1}^{R}\left[h_{0}(n)+e_{i}(n)\right] \\
E\left[e_{i}(n)\right]=\sum_{i} e_{i}(n)=0
\end{array}
$$

The following is an energetic analysis of the relationship between the signal-to-noise ratio of the transfer function after noise reduction and the signal-to-noise ratio of the transfer function derived from a single measurement (single-cycle measurement).

Output for single-cycle measurements:

$$
y_{0}(n)=h_{0}(n)+e_{0}(n)
$$

Signal-to-noise ratio for single-cycle measurements:

$$
S N R=\frac{\left[h_{0}(n)\right]^{2}}{\left[e_{0}(n)\right]^{2}}=\frac{e_{s}^{2}}{e_{n}^{2}}
$$

After noise reduction:

$$
S N R^{\prime}=\frac{\left[R \cdot h_{0}(n)\right]^{2}}{\left[\sum_{i=1}^{R} e_{i}(n)\right]^{2}}=\frac{\left[R \cdot h_{0}(n)\right]^{2}}{\sum_{i=1}^{R}\left[e_{i}(n)\right]^{2}}=\frac{\left[R \cdot h_{0}(n)\right]^{2}}{R \cdot\left[e_{0}(n)\right]^{2}}=R \cdot \frac{e_{s}^{2}}{e_{n}^{2}}
$$

where, $e_{0}(n)$ is the random noise present in the measurement system when the input signal is a single cycle, and $y_{0}(n)$ is the transfer function measured in a single cycle. $e_{s}^{2}$ is the power of the transfer function in the absence of noise, i.e., the signal power; and $e_{n}^{2}$ is the noise power. From the above derivation, it can be seen that the signal-to-noise ratio improves by a factor of $\mathrm{R}$ after R-average noise reduction, because the R-band noises are independent of each other, and the random variables that are independent of each other are the sum of their respective variances; and the signals are correlated, so the signal-to-noise ratio is improved after averaging.

\section{Pool trial validation}

In order to verify the effectiveness of the method in this paper, a validation trial was carried out in an anechoic pool. Three receiving hydrophones are fixed on the shell of the underwater vehicle on both sides, and the hydrophones are led to the receiving device, which performs signal conditioning, filtering, amplification, sampling and storage. Figure 2 shows the reception of the three hydrophones. The time domain transfer function obtained after the signal is processed. 

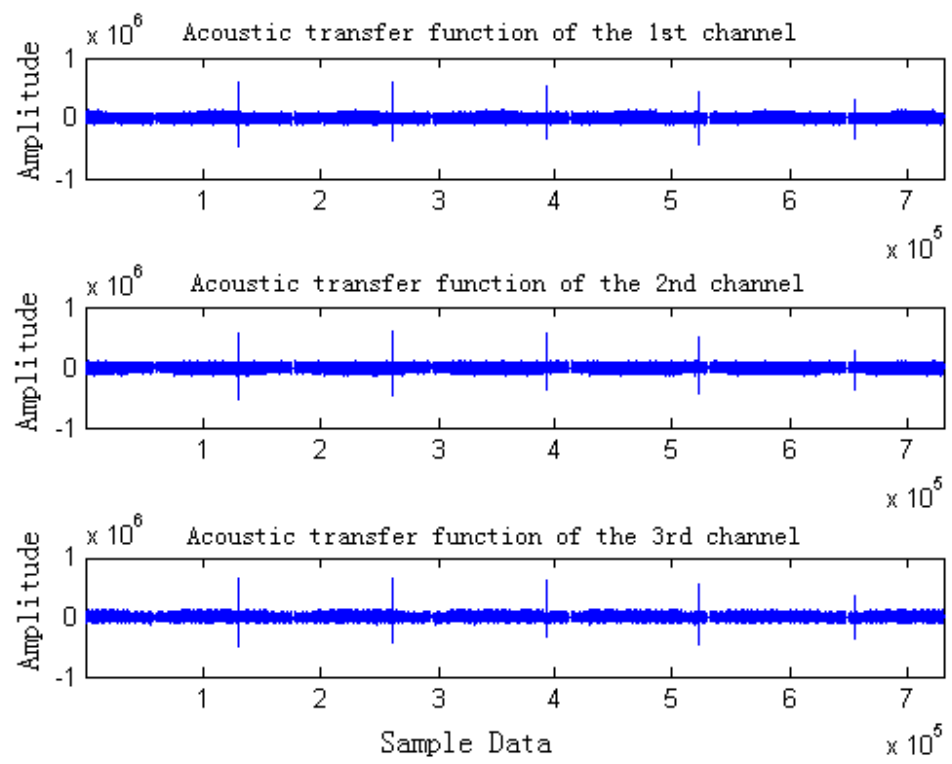

Fig. 2. The signal containing a 5-cycle transfer functions.

Figure 3 is the phase response curve of the transfer function for three consecutive cycles measured by MLS sequence in the anechoic pond, and the phase frequency characteristic curve is observed comparatively. In this paper, the slope of the first period at $1800 \mathrm{~Hz} 2300 \mathrm{~Hz}$ is obtained by the least squares method, and the time difference $\tau$ can be obtained by dividing the slope by $2 \pi$. For the second cycle and subsequent cycles, subtract the time difference of $\tau$ to compensate the phase to the first cycle; then do even symmetry of the real part of the frequency response, odd symmetry of the imaginary part, the final frequency response, that is, the transfer function frequency response after the multi-cycle phase compensation.

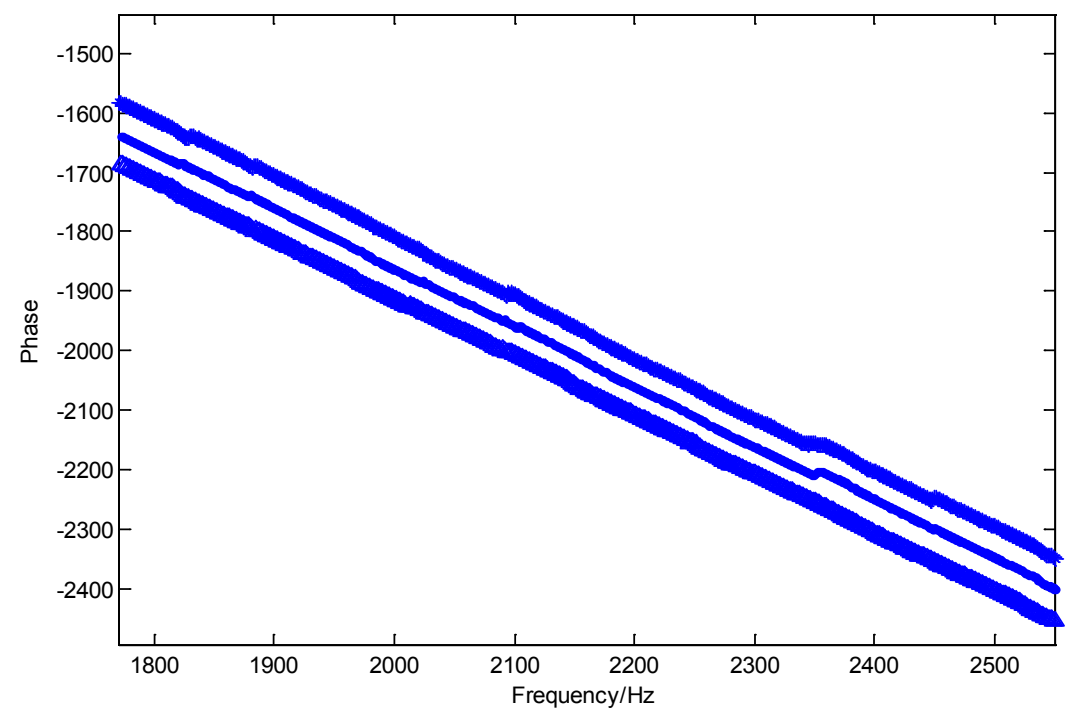

Fig. 3. Phase corresponding curve of the transfer function of first 3 cycles. 


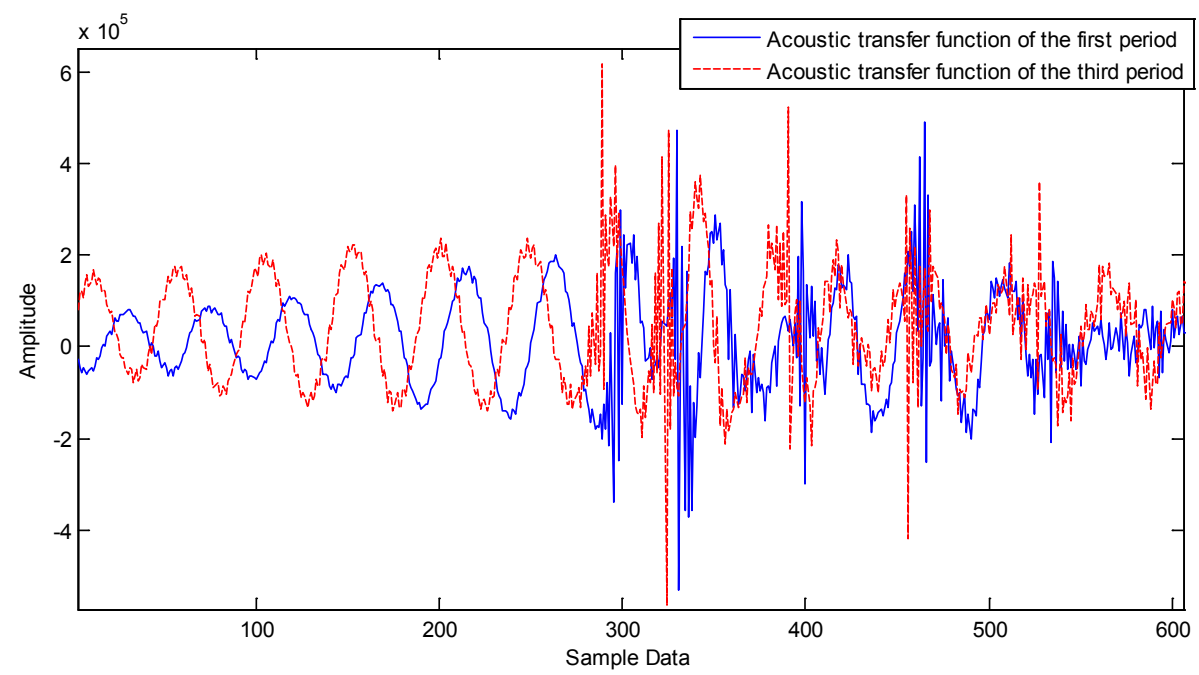

Fig. 4. Local details of the transfer function.

Due to the random characteristics of the noise in the measurement environment, the environmental noise is represented as white spectrum in the frequency spectrum, which can be used to measure the sound scattering characteristics of the underwater vehicle through multiple cycles, and time domain averaging of the signal of each measurement period obtained from the measurement, which will greatly reduce the noise contained in the measurement results and improve the signal-to-noise ratio.
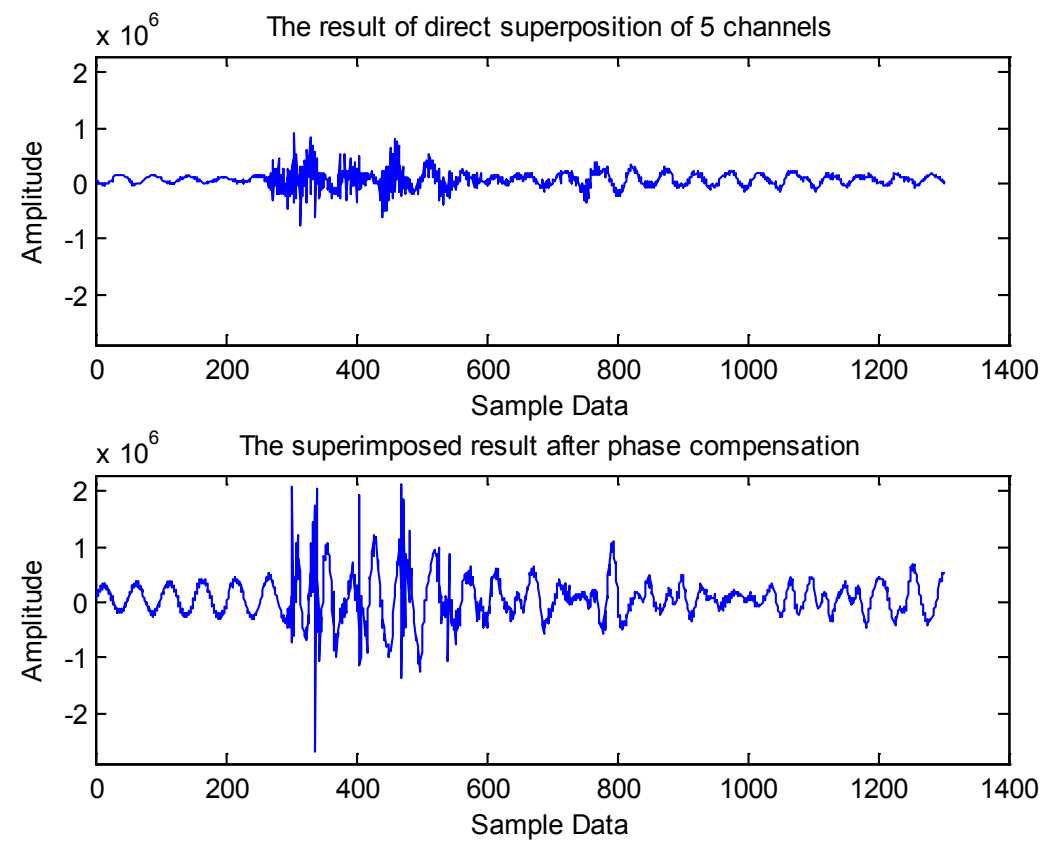

Fig. 5. Comparison of direct overly effect an in-phase overly effect.

The transfer function of the underwater vehicle after noise reduction can be obtained by averaging the five segments after phase compensation in the time domain, as shown in 
Figure 5. Comparing the upper and lower graphs, it can be found that the transfer function after phase compensation and superimposed averaging is relative to the transfer function directly superimposed on the single-cycle measurement and the random noise is obviously reduced. Comparing Figures 4 and 5, it is found that although the amplitude of the waveform can be partially improved by direct averaging, it leads to serious distortion of the transfer function waveform, which greatly changes the properties of the original measured single-cycle transfer function. From the comparative analysis of the two figures, it can be concluded that, relative to the single-period transfer function measurement, the averaging method based on phase compensation for multi-period measurement plays a good noise reduction effect in waveform fidelity at the same time.

\section{Conclusion}

In order to avoid the cumulative effect of phase error through time domain averaging when the transfer function is obtained from the underwater vehicle in the test measurement, this paper proposes a transfer function measurement method based on multi-period transfer function phase compensation for the underwater vehicle, and the anechoic pool test proves that this method reduces the random noise and obtains more realistic time domain average transfer function results, which can effectively improve the signal-to-noise ratio of the underwater vehicle transfer function obtained from the test measurement.

\section{References}

1. Cheng C. I., Wakefield G. H., Introduction to head-related transfer functions (HRTFs): Representations of HRTFs in time, frequency, and space[C].Audio Engineering Society Convention 107. Audio Engineering Society, 1999.

2. Fan Jun, Tang Weilin, Zhuo Linkai. Plate Element Method for Predicting Echo Characteristics of Sonar Targets. Ship mechanics. 2012.

3. Stan G. B., Embrechts J. J., Archambeau D., Comparison of different impulse response measurement techniques[J]. Journal of the Audio Engineering Society, 2002, 50(4): 249-262.

4. RifeD.D.,Vander kooyJ., Transfer-function measurement with maximum-length sequences[J]. Journal of the Audio Engineering Society, 1989, 37(6): 419-444.

5. Vanderkooy J., Aspects of MLS measuring systems[J]. Journal of the Audio Engineering Society, 1994, 42(4): 219-231. 